\title{
Encapsulated Pd Nanocrystals Supported by Nanoline-Structured $\mathrm{SrTiO}_{3}(001)$
}

\author{
Fabien Silly* and Martin R. Castell \\ Department of Materials, University of Oxford, Parks Road, Oxford OX1 3PH, U.K. \\ Received: March 15, 2005; In Final Form: April 18, 2005
}

\begin{abstract}
Palladium nanocrystals were grown on a nanostructured $\mathrm{SrTiO}_{3}(001)$ surface and annealed in ultrahigh vacuum at $620{ }^{\circ} \mathrm{C}$. This leads to the so-called strong metal-support interaction (SMSI) state, characterized by encapsulation of the metal clusters with an oxide layer. Scanning tunneling microscopy (STM) of the oxide adlayer on the $\operatorname{Pd}(111)$ cluster surface reveals two superstructures with different lattice parameters and crystallographic rotations. Interpretation of the STM images is most readily achieved via noncommensurate $\mathrm{TiO}_{x}$ surface layers which result in two distinct Moiré patterns.
\end{abstract}

\section{Introduction}

Metallic nanocrystals on oxide supports display properties that are of interest for both basic and applied research especially in the fields of heterogeneous catalysis and gas sensing. ${ }^{1-3}$ Any interaction between the metallic particles and the support which affects the activity of the system is therefore of fundamental importance. One example is the strong metal-support interaction (SMSI) ${ }^{4}$ where a change in catalytic activity is generally associated with the metal particles being covered by a thin oxide layer. The current understanding is that the metal particle chemically reacts with the support allowing elements from the support to migrate onto the particle and form a suboxide on its surface. ${ }^{5}$

One of the most popular systems for SMSI studies are catalysts composed of noble metals on titania supports. Ti ions migrate onto the noble metal surface ${ }^{6}$ and form a thin $\mathrm{TiO}_{x}$ layer which in turn significantly influences the catalytic activity. ${ }^{7-11}$ Atomic resolution scanning tunneling microscopy (STM) studies of $\mathrm{Pt}^{12,13}$ and $\mathrm{Pd}^{14}$ on $\mathrm{TiO}_{2}$ supports have revealed the complex atomic arrangement of the $\mathrm{TiO}_{x}$ suboxide layer that covers the metals. The oxide layer and the noble metal tend to not have a commensurate interface.

In this paper, we report on STM studies of Pd nanocrystals on $\mathrm{SrTiO}_{3}(001)$ supports and show that encapsulation takes place. $\mathrm{SrTiO}_{3}$ is a cubic perovskite crystal which displays a multitude of reconstructions on its (001) surface depending on sample preparation. Moreover, we have recently observed that extended high temperature annealing under ultrahigh vacuum (UHV) conditions causes epitaxial anatase $\mathrm{TiO}_{2}(001)$ island growth on the $\mathrm{SrTiO}_{3}$ surface. ${ }^{15} \mathrm{Ti}$ ions therefore appear to be sufficiently mobile in reduced $\mathrm{SrTiO}_{3}$ crystals to be able to migrate onto $\mathrm{Pd}$ nanocrystals and encapsulate them with a $\mathrm{TiO}_{x}$ layer. We show that there are two distinct types of decoration layers that appear on the top of the Pd nanocrystals, both of which can be attributed to Moiré patterns.

\section{Experimental Method}

Nb-doped ( $0.5 \%$ weight $) \mathrm{SrTiO}_{3}$ single crystals with epipolished (001) surfaces were supplied by PI-KEM, Surrey, U.K. The high level of $\mathrm{Nb}$ doping resulted in a room temperature resistivity of $10^{-3} \Omega \mathrm{m}$ which increased with increasing sample temperature. The samples were introduced into the ultrahigh vacuum (UHV) chamber of a scanning tunneling microscope (JEOL JSTM4500S) operating at a pressure of $10^{-10}$ mbar. Etched tungsten tips were used to obtain constant current images at room temperature with a bias voltage applied to the sample.

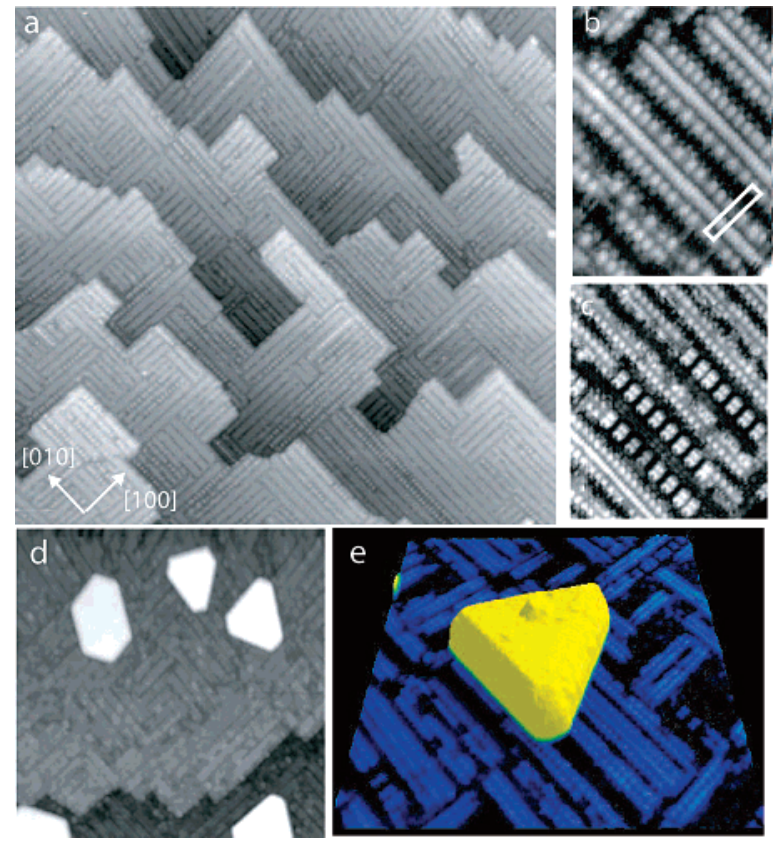

Figure 1. (a) STM image of nanoline-structured $\mathrm{SrTiO}_{3}(001), 157 \times$ $154 \mathrm{~nm}^{2}$. (b) Triple-row nanolines order into a $(9 \times 2)$ surface reconstruction $\left(14 \times 10 \mathrm{~nm}^{2}\right)$. (c) Surface with triple-row nanolines, doublerow nanolines, and nanodots $\left(21 \times 17 \mathrm{~nm}^{2}\right)$. (d) Pd nanocystals on $\mathrm{SrTiO}_{3}(001)\left(80 \times 80 \mathrm{~nm}^{2}\right)$. (e) $3 \mathrm{D}$ representation of a Pd nanocrystal on double-row and triple-row nanolines $\left(V_{\mathrm{s}}=+0.8 \mathrm{~V}, I_{\mathrm{t}}=0.3 \mathrm{nA}\right)$.

Sample heating in the UHV chamber was achieved by passing a current through the sample which allowed anneal temperatures of up to $1400{ }^{\circ} \mathrm{C}$ to be reached. Temperature measurement was performed through a viewport with an optical pyrometer. $\mathrm{SrTiO}_{3}$ samples were annealed in UHV at temperatures between 600 and $800{ }^{\circ} \mathrm{C}$ typically for $30 \mathrm{~min}$ to remove contamination. The surface was then sputtered with argon ions and subsequently annealed in UHV at $875^{\circ} \mathrm{C}$ for $10 \mathrm{~h}$. Pd was deposited from an e-beam evaporator (Oxford Applied Research EGN4) using 99.95\% pure Pd rods supplied by Goodfellow UK. The $\mathrm{SrTiO}_{3}$ substrate temperature during $\mathrm{Pd}$ deposition was at $620^{\circ} \mathrm{C}$. Following Pd deposition, the samples were annealed for $1 \mathrm{~h}$ at 620 ${ }^{\circ} \mathrm{C}$.

\section{Results}

A. Palladium Nanocrystal Growth on Nanoline-Structured $\mathrm{SrTiO}_{3}(\mathbf{0 0 1})$. Figure 1a shows the $\mathrm{SrTiO}_{3}(001)$ surface after a 

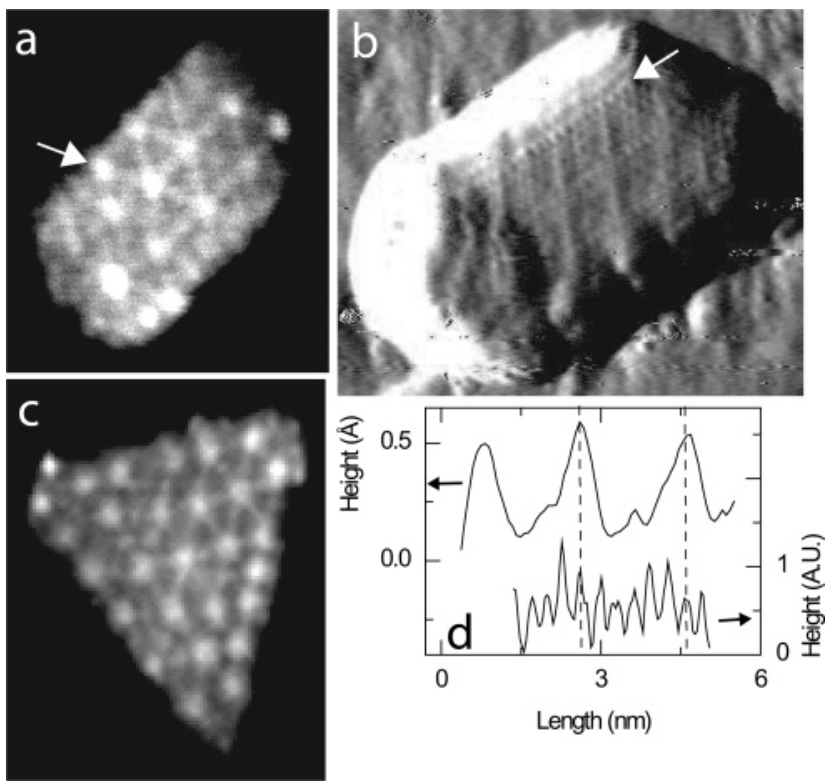

Figure 2. Encapsulated $\mathrm{Pd}$ nanocystals on $\mathrm{SrTiO}_{3}(001)$ with a wagon wheel (structure A) termination. Image sizes: (a) $12 \times 14 \mathrm{~nm}^{2}$, (b) 13 $\times 10 \mathrm{~nm}^{2}$, and (c) $21 \times 22 \mathrm{~nm}^{2}$. (d) Profiles taken along the arrow in parts a (top curve) and $\mathrm{b}$ (bottom curve) $\left(V_{\mathrm{s}}=+0.8 \mathrm{~V}, I_{\mathrm{t}}=0.3 \mathrm{nA}\right)$.

sputtering cycle and annealing in UHV for $10 \mathrm{~h}$ at $875^{\circ} \mathrm{C}$. The surface is fully covered by nanoline domains. The nanostructures covering the surface are composed of domains of double-row nanolines and nanodots (Figure 1c), as previously reported in ref 16 , but in addition, this sample also has domains of triplerow nanolines (Figure 1b). The corrugation along the central row of the triple line and along the external rows is 4.0 and 8.0 $\AA$, respectively, corresponding to single and double factors of the unit cell of $\mathrm{SrTiO}_{3}$, respectively. The closest packing of triple-row nanolines gives rise to domains with a $(9 \times 2)$ reconstruction of the $\mathrm{SrTiO}_{3}$ surface (Figure 1b). In contrast, the double-row domains give rise to $(6 \times 2)$ ordering (Figure $1 \mathrm{c}$ and ref 16). Palladium was deposited on nanoline-structured $\mathrm{SrTiO}_{3}(001)$ at a substrate temperature of $620{ }^{\circ} \mathrm{C}$ with a subsequent anneal at $620^{\circ} \mathrm{C}$ for $1 \mathrm{~h}$. Figure $1 \mathrm{~d}$,e shows that this treatment gives rise to truncated triangle Pd clusters. The shape of the nanocrystal is characteristic of a (111) plane top facet, with three large (111) side facets and three small (001) side facets. The interface is a (111) plane, described in formal terms as $(111)_{\mathrm{Pd}}\left\|(001)_{\mathrm{SrTiO}_{3}},[110]_{\mathrm{Pd}}\right\|[100]_{\mathrm{SrTiO}_{3}}$, giving rise to coincidence epitaxy. For a typical deposition and anneal cycle, the average cluster height is $1.4 \mathrm{~nm}$ and the width is $15 \mathrm{~nm}$. Our study of how the surface reconstruction and substrate temperature affect the Pd nanocrystal shape is reported in ref 17 .

B. Palladium Nanocrystal Surfaces: The Wagon Wheel Superstructure. Figure 2 shows STM images of the (111) top surface of some of the Pd clusters. A superstructure, which we will call structure A, consisting of bright spots connected by lines is visible (Figure $2 \mathrm{a}-\mathrm{c}$ ). This superstructure has been previously termed the "wagon wheel" structure. The unit cell of the superstructure is nearly hexagonal with a $19.8 \pm 0.6 \AA$ lattice parameter (top graph in Figure 2d). The bright spots are not always perfectly hexagonally distributed on large cluster surfaces, revealing imperfect ordering of the superstructure (Figure 2c). In Figure 2b, an atomically resolved STM image is shown of the top surface of the nanocrystal. Besides the wagon wheel structure, an additional periodicity of $3.2 \pm 0.1$ $\AA$ can be observed (bottom graph in Figure 2d). The atoms are aligned along the line connecting the bright spots of the wagon wheel structure.

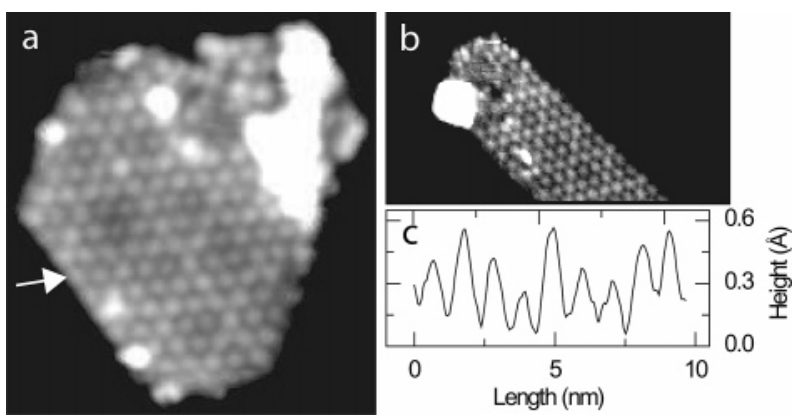

Figure 3. Encapsulated $\mathrm{Pd}$ nanocystals on $\mathrm{SrTiO}_{3}(001)$ with a structure B termination: (a) STM image $19 \times 18 \mathrm{~nm}^{2}$; (b) STM image $26 \times 15$ $\mathrm{nm}^{2} ;(\mathrm{c})$ cross section taken along the arrow in part a $\left(V_{\mathrm{s}}=+0.8 \mathrm{~V}, I_{\mathrm{t}}\right.$ $=0.3 \mathrm{nA})$.

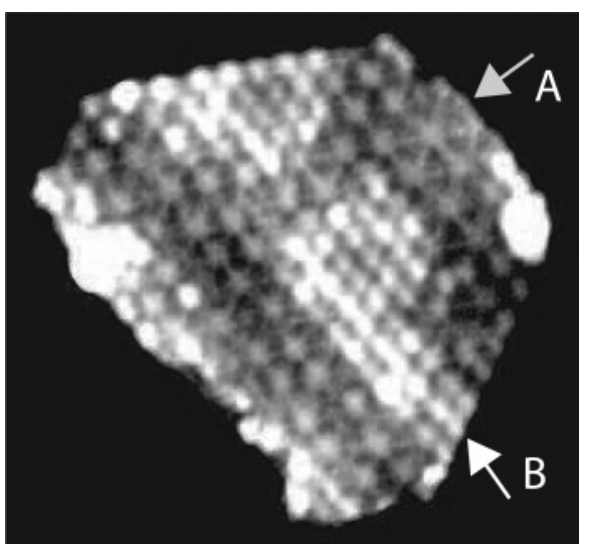

Figure 4. Encapsulated $\mathrm{Pd}$ nanocystal on $\mathrm{SrTiO}_{3}(001)$ with a mixed structure $\mathrm{A}$ and $\mathrm{B}$ termination. The structure $\mathrm{A}$ and $\mathrm{B}$ domains are indicated by arrows $\left(21 \times 22 \mathrm{~nm}^{2}\right)\left(V_{\mathrm{s}}=+0.8 \mathrm{~V}, I_{\mathrm{t}}=0.3 \mathrm{nA}\right)$.

C. Palladium Nanocrystal Surfaces: The Hexagonal Superstructure. A second superstructure, which we will call structure B, was observed on the top of other clusters, as shown in Figure 3. This superstructure is nearly perfectly hexagonal with a $10.5 \pm 0.2 \AA$ lattice parameter. Every third line of spots appears bright compared to the others (Figure 3a,b).

As shown in Figure 4, superstructures A and B can coexist on the same cluster with no step edges separating them. Careful investigation of Figure 4 shows that structures $\mathrm{A}$ and $\mathrm{B}$ are not aligned but that there is an $\sim 6^{\circ}$ orientation difference between the two types of superlattices. On average, structure B appears $\sim 0.3 \AA$ A higher than structure A.

\section{Discussion}

Figure $2 \mathrm{~b}$ shows that the Pd clusters are covered by an adlayer with hexagonal symmetry and a $3.20 \AA$ atomic periodicity. This does not match the $\operatorname{Pd}(111)$ periodicity $(2.75 \AA)$ or any of the $\mathrm{SrTiO}_{3}(111)$ reconstructions. Bennett et al. ${ }^{14}$ have shown that a $\mathrm{Pd}$ film grown on $\mathrm{TiO}_{2}$ at high temperatures is covered by a $\mathrm{TiO}_{x}$ layer with a $3.3 \AA$ periodicity. This layer periodicity is very close to the $3.20 \AA$ periodicity observed on the $\mathrm{Pd}$ nanocrystals grown on our $\mathrm{SrTiO}_{3}$ substrates. Moreover, it has recently been shown that under annealing conditions anatase $\mathrm{TiO}_{2}$ islands can spontaneously grow on this nanostructured surface, ${ }^{15}$ indicating that $\mathrm{Ti}$ and $\mathrm{O}$ adatoms are highly mobile on the $\mathrm{SrTiO}_{3}(001)$ surface at elevated temperatures. Therefore, it is very likely that a $\mathrm{TiO}_{x}$ layer encapsulates the Pd nanocrystals on $\mathrm{SrTiO}_{3}$ in a similar way to the encapsulation of $\mathrm{Pd}$ on $\mathrm{TiO}_{2}$. This could be verified through further experiments on the encapsulated Pd nanocrystals using X-ray photoelectron spectroscopy, low energy ion scattering, and scanning tunneling 
spectroscopy which would allow us to better determine the physical and chemical properties of the encapsulation layer.

In the $\mathrm{Pd}$ on $\mathrm{TiO}_{2}$ experiments, ${ }^{14}$ the adlayer was assigned to be composed of a hexagonal $\mathrm{O}$ layer (the $\mathrm{O}$ adatoms have the same periodicity and orientation as the underlying Pd layer) sandwiched between the Pd and the outermost Ti layer. This arrangement of atoms is reminiscent of the $\mathrm{TiO}(111)$ surface which has an atomic periodicity of $3.0 \AA$.

We will now discuss how the superposition of two layers having different periodicities can affect STM image contrast. The superposition of two lattices will result in the formation of a Moiré pattern. An algebraic approach can be used to calculate the points of coincidence for these two lattices. The aim is to find a structural model for the $\mathrm{O}$ on the $\mathrm{Pd}(111)$ lattice and the $\mathrm{Ti}$ adlayer which is consistent with the distances, the angles, and the translational symmetry seen in the STM images. The condition of coincidence of the two superposed lattices is governed by the following equation: ${ }^{18}$

$$
\left(\begin{array}{ll}
s & \frac{1}{2} s \\
0 & \frac{\sqrt{3}}{2} s
\end{array}\right)\left(\begin{array}{l}
h \\
k
\end{array}\right)=\left(\begin{array}{ll}
\cos \theta & -\sin \theta \\
\sin \theta & \cos \theta
\end{array}\right)\left(\begin{array}{ll}
a & \frac{1}{2} a \\
0 & \frac{\sqrt{3}}{2} a
\end{array}\right)\left(\begin{array}{l}
m \\
n
\end{array}\right)
$$

where $s$ and $a$ denote the interatomic distances in the substrate layer and adsorbate layer, respectively. $\theta$ is the angle between the substrate and the adlayer. $h, k$, and $m, n$ are integer multiples of the $\mathrm{O}(s)$ and the $\mathrm{Ti}(a)$ interatomic spacing which lead to the coincidence between the lattices. Equation 1 can be solved for the variables $a$ and $\theta$ :

$$
\begin{aligned}
& \theta=\arctan \left(\frac{\sqrt{3}(k m-h n)}{2 h m+h n+k m+2 k n}\right) \\
& a=s \sqrt{\frac{h^{2}+h k+k^{2}}{m^{2}+m n+n^{2}}}
\end{aligned}
$$

From eq 1, we can also determine the coincidence periodicity $r$ (Moiré periodicity) and angle $\phi$ of the Moiré pattern with respect to the substrate.

$$
\begin{aligned}
& r=\frac{s}{2} \sqrt{(2 h+k)^{2}+3 k^{2}} \\
& \phi=\arctan \left(\frac{\sqrt{3} k}{2 h+k}\right)
\end{aligned}
$$

Possible solutions for eq 1 were obtained by iteration for various $h, k, m, n$ multipliers with $s$ fixed at $2.75 \AA$. For each set of these multipliers, the values for the interatomic adlayer distance $a$, the coincidence periodicity $r$, the Moiré angle $\phi$, and the adlayer angle $\theta$ were calculated. By variation of all integer multipliers from 0 to 40 , only those sets which are compatible with the experimental values of $a(\sim 3.20 \AA), r$, and $\phi$ were considered for further analysis.

Structure A (the wagon wheel structure) has a measured periodicity of $19.8 \pm 0.6 \AA$ and is not rotated with respect to the Pd orientation. A Moiré pattern with these features can be reproduced with the indices $h=7, k=0, m=6, n=0, s_{\mathrm{A}}=$ $2.75 \AA, \theta_{\mathrm{A}}=0^{\circ}$, and $a_{\mathrm{A}}=3.21 \AA$. The calculated periodicity of the Moiré pattern using the parameters above is $r_{\mathrm{A}}=19.25$ $\AA$ which is close to the measured value $19.8 \AA$. Figure 5 shows this Moiré pattern schematically. A hexagonal adlayer of 3.21 A periodicity representing Ti atoms (blue balls) is superimposed on a hexagonal layer of $2.75 \AA$ periodicity representing O atoms (red balls). This forms a $\mathrm{TiO}_{1.36}$ layer. The $\mathrm{Ti}$ and $\mathrm{O}$ sublattices

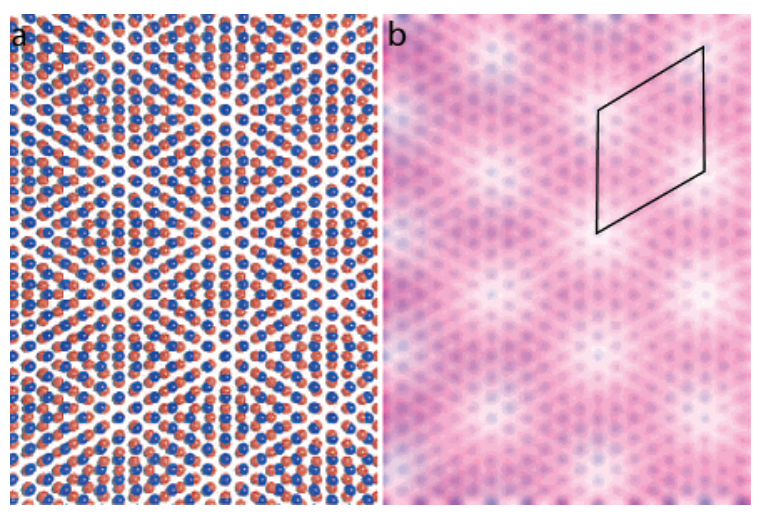

Figure 5. Model for the wagon wheel structure (A): (a) A layer of $O$ is placed on the Pd 3-fold hollow sites (either all fcc or all hep sites) with exactly the same lattice spacing $(2.75 \AA$ ). On top of the O layer, a Ti layer is added with a lattice spacing of $3.21 \AA$. The red spheres are $\mathrm{O}$ atoms, and the blue spheres are Ti atoms. (b) As an aid to STM image interpretation, model a was blurred and a unit cell of $19.25 \AA$ was added.

have the same orientation $\left(\theta_{\mathrm{A}}=0^{\circ}\right)$ which results in a Moiré pattern that has a $19.25 \AA$ periodicity.

The superposition of the $\mathrm{Ti}$ and $\mathrm{O}$ sublattices will give rise to topographic variations. This is because a singly coordinated $\mathrm{Ti}$ atom located directly above an $\mathrm{O}$ atom will stand proud further from the surface than a 3-fold coordinated $\mathrm{Ti}$ atom located in a hollow site. A 2-fold coordinated Ti atom will be located at an intermediate height. We selected a way of representing the calculated Moiré pattern (Figure 5) to show the height variation: bright regions are high, and dark regions are low as in STM images. We can therefore interpret the STM images in Figure 2 in the following manner: the bright spots are singly coordinated $\mathrm{Ti}$ atoms, the lines connecting the bright spots are 2 -fold coordinated $\mathrm{Ti}$ atoms, and the dark centers of the triangles are 3-fold coordinated $\mathrm{Ti}$ atoms. Of course, this simple geometric procedure is not a true STM image simulation, since electronic effects are neglected.

The Moiré pattern with a $10.5 \AA$ periodicity, as observed in structure B, cannot be accurately reproduced with a $\sim 3.2 \AA$ periodicity adlayer superimposed on a $2.75 \AA$ periodicity substrate. However, the STM image in Figure 3a shows that every third row of spots appears brighter than the other two rows. We therefore explored Moiré patterns with $3 \times 10.5 \AA$ periodicities, that is, $31.5 \AA$. We selected the indices $h=11, k$ $=1, m=3$, and $n=8$, which lead to a misfit orientation of $\theta_{\mathrm{B}}$ $=19.602^{\circ}$ and a Ti adlayer periodicity of $a_{\mathrm{B}}=3.22 \AA$. The Moiré periodicity and angle are calculated to be $r_{\mathrm{B}}=31.715 \AA$ and $\phi_{\mathrm{B}}=4.3^{\circ}$.

Figure 6 shows a schematic representation of a hexagonal adlayer of $3.22 \AA$ periodicity representing Ti atoms (blue balls) superimposed on an hexagonal layer of $2.75 \AA$ periodicity representing $\mathrm{O}$ atoms (red balls). This represents a $\mathrm{TiO}_{1.37}$ adlayer that covers the Pd nanocrystals. The same image blurring treatment as that applied in Figure $5 \mathrm{~b}$ is shown in Figure $6 \mathrm{~b}$. It can now be readily seen that, although the true periodicity of the Moiré pattern is $31.715 \AA$, the periodicity that appears most dominant in the pattern is $10.57 \AA$. Both unit cells are shown in Figure 6b. As explained previously, the brighter the regions in the pattern, the lower the coordination of the Ti ions. This model is in very good agreement with the STM images of the superstucture shown in Figure 3.

The STM image in Figure 4 shows that Moiré structures A and $\mathrm{B}$ can coexist on the same Pd island. The stoichiometries of structures $\mathrm{A}$ and $\mathrm{B}$ are $\mathrm{TiO}_{1.36}$ and $\mathrm{TiO}_{1.37}$, respectively. Given 


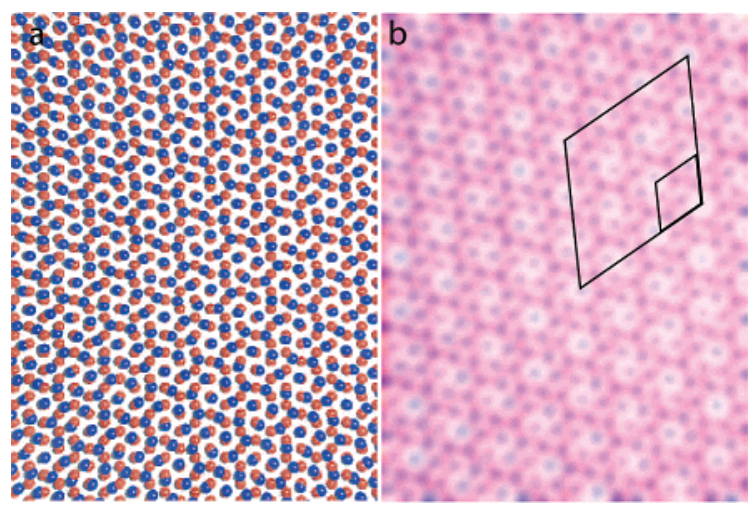

Figure 6. Model for structure B: (a) A layer of $\mathrm{O}$ is placed on the $\mathrm{Pd}$ 3-fold hollow sites (either all fcc or all hcp sites) with exactly the same lattice spacing $(2.75 \AA)$. On top of the O layer, a Ti layer is added with a lattice spacing of $3.22 \AA$ and rotated at $19.6^{\circ}$ to the $O$ lattice. The red spheres are $\mathrm{O}$ atoms, and the blue spheres are $\mathrm{Ti}$ atoms. (b) As an aid to STM image interpretation, model a was blurred and two unit cells were added. The large $31.7 \AA$ cell is the true Moiré periodicity, and the small $10.6 \AA$ cell is the dominant periodicity seen in the STM images.

this similarity, it cannot be the stoichiometry that is responsible for the coexistence of the two structures. The most obvious explanation is that structures A and B have very similar surface energies and can therefore readily coexist in close proximity.

\section{Conclusion}

We have prepared two new SMSI states for Pd nanocrystals supported on nanostructured $\mathrm{SrTiO}_{3}(001)$. Two distinct Moiré patterns with different lattice periodicities were observed on the top surfaces of the crystals. Atomically resolved STM images can be simulated by encapsulating the Pd nanocrystals with $\mathrm{TiO}_{1.36}$ or $\mathrm{TiO}_{1.37}$ layers. By variation of the adlayer orientation with respect to the Pd lattice, two Moiré patterns can be created that match the images observed by STM. These experiments show that $\mathrm{Pd}(111)$ islands on nanostructured $\mathrm{SrTiO}_{3}(001)$ give rise to different Moiré patterns than have been seen on $\operatorname{Pd}(111)$ islands and thin films on $\mathrm{TiO}_{2}(110)-(1 \times 2)$ substrates. ${ }^{14}$

Acknowledgment. The authors would like to thank the Royal Society and DSTL for funding and Chris Spencer (JEOL UK) for valuable technical support. We are also grateful to Nicolas Weiss (IBM Almaden) and Andrew Powell for helpful discussions.

\section{References and Notes}

(1) Johanek, V.; Laurin, M.; Grant, A. W.; Kasemo, B.; Henry, C. R.; Libuda, J. Science 2004, 304, 16391.

(2) Prevot, G.; Meerson, O.; Piccolo L.; Henry, C. R. J. Phys.: Condens. Mater 2002, 14, 4251.

(3) Prevot, G.; Henry, C. R. J. Phys. Chem B 2002, 106, 12191.

(4) Tauster, S. J.; Fung, S. C.; Garten, R. L. J. Am. Chem. Soc. 1978, 100,170 .

(5) Haller, G. L. J. Catal. 2003, 216, 12.

(6) Datye, A. K.; Kalakkad, D. S. J. Catal. 1995, 155, 148.

(7) Tauster, S. J. ACS Symp. Ser. 1986, $298,1$.

(8) Tanner, R. E.; Liang, Y.; Altman, E. I. Surf. Sci. 2002, 506, 251.

(9) Li, Y. Z.; Xu, B. L.; Fan, Y. N.; Feng, N. Y.; Qiu, A. D.; He, J. M.

J.; Yan, H. P.; Chen, Y. J. Mol. Catal. A: Chem. 2004, 216, 107.

(10) Ito, K.; Tomino, T.; Ohshima, M.; Kurokawa, H.; Sugiyama, K.; Miura, H. Appl. Catal., A 2003, 249, 19.

(11) Chen, W.; Cameron, S.; Gothelid, M.; Hammar, M.; Paul, J. J. Phys. Chem. 1995, 99, 12892.

(12) Dulub, O.; Hebenstreit, W.; Diebold, U. Phys. Rev. Lett. 2000, 84, 3646.

(13) Berko, A.; Szoko, S.; Solymosi, F. Surf. Sci. 2003, 532, 390

(14) Bennett, R. A.; Pang, C. L.; Perkins, N.; Smith, R. D.; Morrall, P.; Kvon, R. I.; Bowker, M. J. Phys. Chem B 2002, 106, 4688.

(15) Silly F.; Castell, M. R. Appl. Phys. Lett. 2004, 85, 3223.

(16) Castell, M. R. Surf. Sci. 2002, 516, 33.

(17) Silly F.; Castell, M. R. Phys. Rev. Lett. 2005, 94, 046103.

(18) Hommrich, J.; Humann S.; Wandelt, K. Faraday Discuss. 2002, $121,129$. 